\title{
A consideration of the effects of the slip displacement on fretting fatigue behaviour
}

\author{
D Dini and D A Hills* \\ Department of Engineering Science, University of Oxford, UK
}

\begin{abstract}
In this paper the possibility is explored that the slip displacement has an influence on the range of stresses experienced by a fretting contact. It is shown that this can have a first-order effect only for a sliding contact.
\end{abstract}

Keywords: partial slip, fretting fatigue, contact problems

\section{INTRODUCTION}

The underlying mechanics describing fretting fatigue are gradually being explained and the processes involved more thoroughly understood. If fatigue as a whole is split up into its elements, the following emerge as key components, but first an analysis of the contact itself will be given. In many problems it is known that the contact problem is difficult to solve and, even with numerical methods such as finite elements, the steepness of the stress gradients present means that great mesh refinement, with attendant demands on computing power, is needed. Some fretting contacts are in sliding, and others in partial slip; the latter can also prove a difficult problem to unravel and again great care is needed. However, the governing algorithms are well understood $[\mathbf{1}, \mathbf{2}]$ and the recent theorem by Ciavarella and Jäger [3, 4] has enabled the repertoire of partial slip solutions, within the scope of half-plane formulations, to be extended significantly. The result of fretting fatigue is, of course, to produce a crack, and its propagation is again well understood; it is known that there is no fundamental difference between a crack of finite size undergoing plain fatigue and one undergoing fretting fatigue. It is simply that additional care is needed in the evaluation of the crack tip stress intensity factor, again because the local stress gradients are so steep. However, when this is done, any crack propagation 'law' may be employed. It is the intermediate step between these two phases of the solution, viz. the initiation of the crack, that is, as expected, proving elusive to explain. Even here, some progress has been made; the critical plane approach has been used with some success

The MS was received on 16 May 2003 and was accepted after revision for publication on 8 September 2003.

* Corresponding author: Department of Engineering Science, University of Oxford, Parks Road, Oxford OX1 3PJ, UK.
[5-7], short crack self-arrest type methods have been developed $[\mathbf{8}, \mathbf{9}]$ and, in some circumstances, the notion of a 'process zone' characterized by an asymptotic local stress field has met with some success [10-12]. Despite these inroads, no completely satisfactory theory has been advanced and verified, and the question of the role of the interface itself is drawn into question.

The effect of an interface on the initiation process is still an open question. If there are two sites for crack nucleation, one in a monolithic component and the other in a contacting pair but with an adhered interface and identical state of stress at the critical point, will the second have a greater propensity to nucleate cracks, because of the influence of asperities? Further, if the interface slips, will the tendency to nucleate cracks be enhanced further? In this paper an element in this edifice is presented, by looking specifically at one influence of slip displacement on nucleation. This is a fundamental question, because, if it is believed that the primary variable controlling crack propagation is the state of stress and that nucleation is also controlled by the range of stress suffered, there is no clear reason why the range of slip displacement present should be relevant. A much-quoted series of papers by Nishioka and Hirakawa [13], dating back to the late 1960s, states that the most damaging range of slip displacement is of the order of $5-50 \mu \mathrm{m}$. It is argued that the damage caused by slip would normally be expected to increase with slip displacement, but that, at higher displacements, wear 'grinds away' embryo cracks, actually increasing the contact's life (see also reference [14]). There does not seem to have been much more recent corroboration of this result (the only intensive investigation of fretting wear and its dependence on slip displacements has been carried out in references [15] to [17]) and it is extremely difficult to devise tests in which slip displacements of this order or less may be applied and varied, but with 
other variables in the contact kept the same. Indeed, it would seem practically impossible to devise any form of a partial slip experiment in which only the slip displacement was varied.

\section{PHYSICAL BASIS}

Here, the effect of slip displacement on the range of stress experienced by any particle on the surface is explored. This will be done for both a sliding contact and one in partial slip using an indenter that has a flat face with rounded corners. However, first the phenomenon using the Hertzian contact as a vehicle will be illustrated. Figure 1 depicts three pairs of bodies having the same relative curvature and therefore, within the Hertz assumption, the same contact pressure and hence state of stress, even if a shearing force is applied. However, when a small rigid-body tangential displacement is imposed, the stress history experienced by points adjacent to the contact on the surface of these contacting pairs experiences quite different effects: in problem (a) the contact is stationary in the curved upper body (1), but displaces with the contact in the half-plane (body 2 ), while in problem (c) the contact is stationary in the lower curved body (2) but displaces in the upper halfplane (body 1) and, lastly, in problem (b) with both bodies curved, the imposition of a small relative tangential displacement causes a shift in the contact in both elements. This effect is not normally allowed for in fretting-type problems, where it is habitually assumed that the contact is fixed in both bodies, at least under partial slip conditions [2], but it clearly means that a particle near the surface of a body where the contacting body exhibits a relative displacement must experience a greater stress range than one where the contacting body is spatially fixed. It is sometimes suggested that the effect described lies outside the linear theory of elasticity, but this is not so when a slipping interface is present, and this remark applies a fortiori if the bodies are sliding.

Figure 2 depicts a contacting pair where body 2 is a half-plane and body 1 an elastically similar punch, the latter having a flat face of width $2 a$ and with ends of radius $R$. It is assumed that, when the normal load, $P$, is applied, the contact extends over an interval $2 b$ and that this is sufficiently small compared with $2(a+R)$ for half-plane theory to be applied. This geometry, originally studied by Scthayerman [18], has recently received a lot of attention, at least partly because of its relevance to the gas turbine fan-blade dovetail, and there are now several papers in English describing its properties, both under normal loading with sliding and certain partial slip conditions. This geometry provides the ideal vehicle to study the influence of slip displacement on stress range because, from the practical viewpoint, it has relevance to dovetails and other nearly
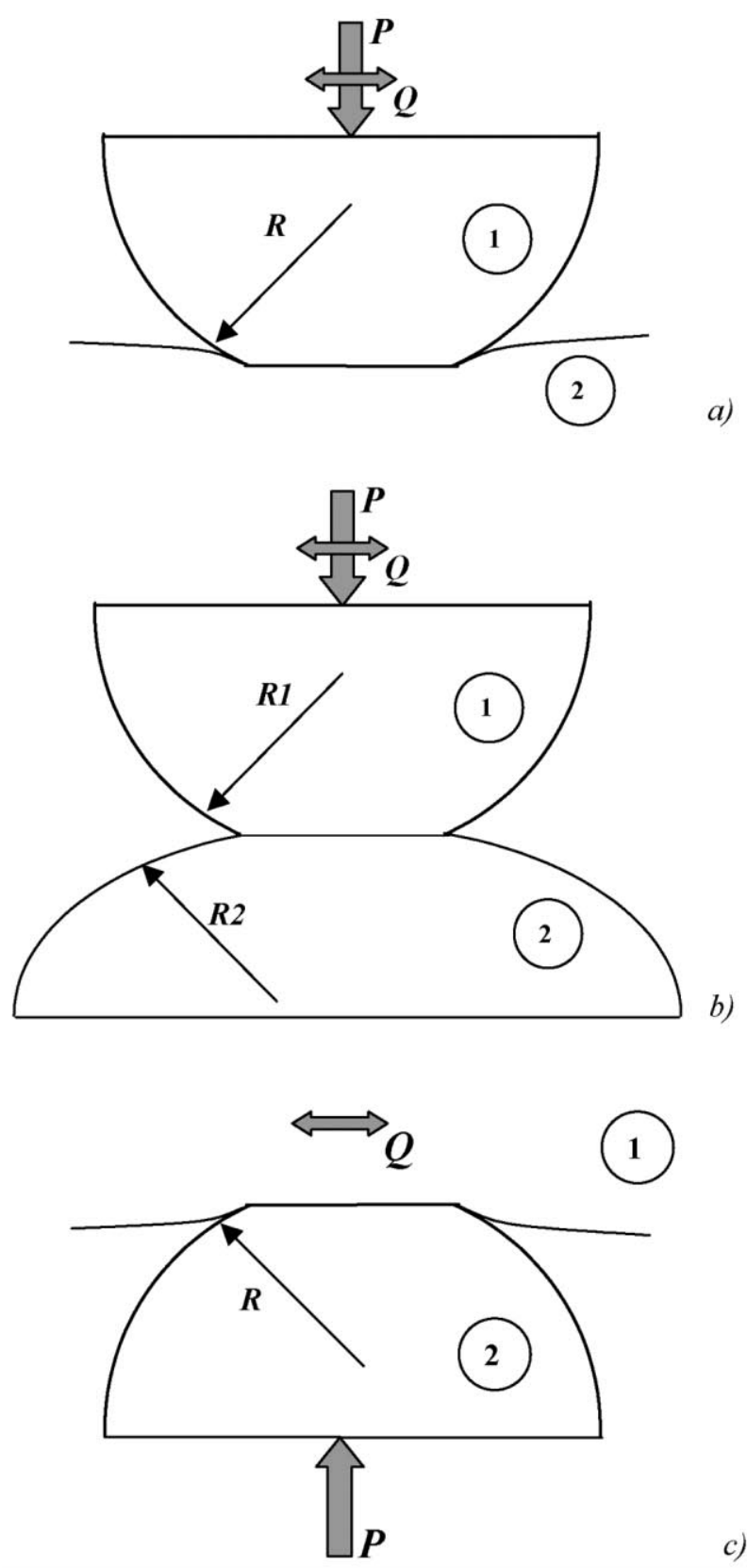

c)

Fig. 1 Hertzian contact problems, showing the effect of curvature on slip displacement experienced: (a) fixed in body 1, (b) moving in both and (c) fixed in body 2

complete contact problems and, from the analytical standpoint, because the severest contact pressure, though finite, is localized in a very small region near the contact edge. It follows that this class of problem is much more likely to exhibit a displacement or 'convection' effect than the Hertzian contact.

Both sliding and partial slip contacts will be analysed in the following sections in order to study the effects of displacements on the local variation of the stress field in different loading conditions. The results and the calculations proposed will all refer to contacts undergoing fully reversing loading cycles and characterized by a coefficient of friction $f=0.65$. 


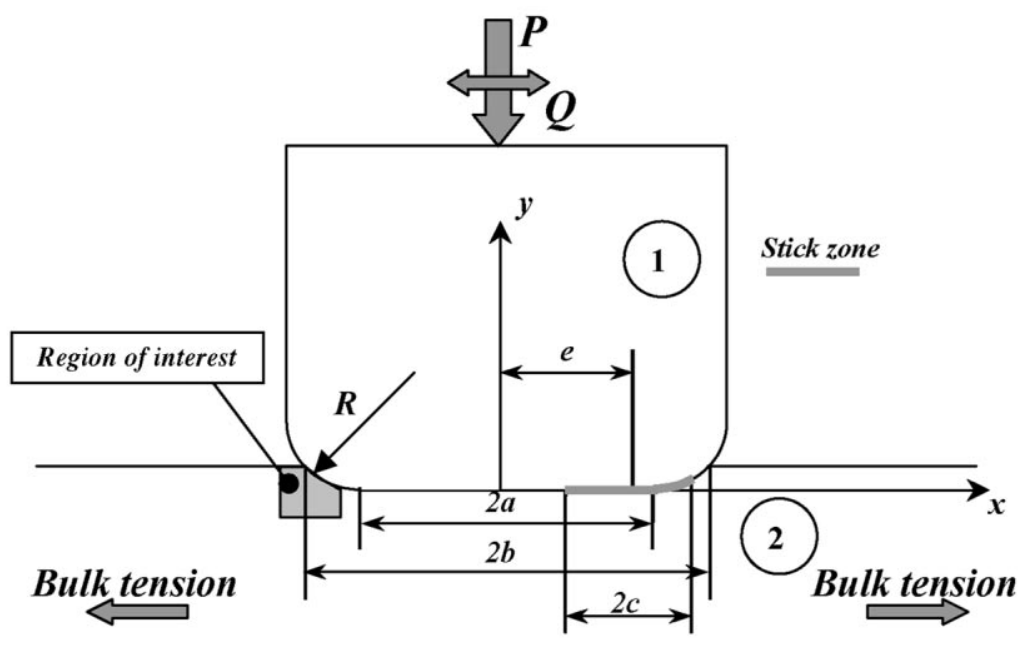

Fig. 2 Flat and rounded contacts. The shadowed region is the domain of interest in Fig. 7

\section{PARTIAL SLIP CONTACT}

Problems involving partial slip imply, by definition, that there is at least one pair of corresponding surface particles in the two contacting bodies where there is no relative slip, i.e. at least one pair of particles is always adhered. It follows that the maximum slip displacement that arises is limited by the compliance of the material (in the sense of its elastic constants) and the compliance of the contact. The present authors have investigated the slip displacement present in a 'flat and rounded' contact when there is both a shear force and bulk tension present, so that the stick region is small compared to the overall contact and attached to the leading edge. The largest values of slip displacement then arise at the trailing edge of the contact.

In order to investigate the relative displacement of the contacting surfaces, the partial slip contact problem itself needs to be solved. For the case when the contacting bodies are elastically similar, the slip displacement, $g(x)$, is related to the shearing traction distribution, $q(x)$, by

$$
\frac{1}{A} \frac{\partial g(x)}{\partial x}=\frac{1}{\pi} \int \frac{q(\xi) \mathrm{d} \xi}{x-\xi}
$$

where $A$ is the composite compliance defined as

$$
A=2 \frac{k+1}{4 \mu}
$$

with $k=3-4 \nu$ in plane strain, $\nu$ the Poisson's ratio and $\mu$ the modulus of rigidity of the material. In the flat and rounded contact problem, problems involving the simultaneous application of a shearing force and remote bulk tension require numerical solution and are partly but not fully covered by the literature (see references [19] to [21]). This regime is of particular interest here because it is possible to have a very small stick region attached to one end of the contact, thereby giving the maximum amount of 'convection', but it does demand a numerical solution. A brief summary of the technique used to solve the partial slip problem will be presented here.

Remote bulk tension is applied to body 2 (Fig. 2), and it is assumed that the stick zone is of semi-width $c$, centred at $e$. Consider the case when $e+c \leqslant b$ so that there are generally two slip zones at the edge of the contact (or one slip zone at the edge of the contact and a stick zone attached to the leading edge in the limit case, i.e. when $e+c=b$ ). The shear traction distribution can be written as

$$
\begin{aligned}
& q(x)=f|p(x)| \operatorname{sign}(x), \quad c \leqslant|x-e| \leqslant b(\operatorname{slip}) \\
& q(x)=q_{\text {stick }}(x), \quad|x-e| \leqslant c \text { (stick) }
\end{aligned}
$$

where $\operatorname{sign}(x)$ has to be considered only in the case of reverse (see reference [2]) slip at the leading edge of the contact. In the presence of bulk stress, $\sigma_{0}$, the general integral equation (1) becomes

$$
\frac{1}{\pi} \int_{\text {contact }} \frac{q(\xi) \mathrm{d} \xi}{x-\xi}=-\frac{\sigma_{0}}{4}, \quad|x-e| \leqslant c
$$

By substituting equations (3) and (4) in equation (5), the integral equation governing the specific problem can be derived where the function $G\left(x, \sigma_{0}, c, e\right)$ is known:

$$
\begin{aligned}
\int_{e-c}^{e+c} \frac{q_{\text {stick }}(\xi) \mathrm{d} \xi}{x-\xi}= & -\frac{\sigma_{0} \pi}{4}-\int_{e+c}^{b} \frac{f p(\xi) \mathrm{d} \xi}{x-\xi} \\
& \pm \int_{-b}^{e-c} \frac{f p(\xi) \mathrm{d} \xi}{x-\xi} \\
& \equiv G\left(x, \sigma_{0}, c, e\right), \quad|x-e| \leqslant c
\end{aligned}
$$

The positive sign in front of the right-hand integral is chosen when there is reverse slip in the interval $-b \leqslant x \leqslant e-c$ and the negative sign when there is forward slip in this interval. Equation (6) can then be normalized by setting $s=(x-e) / c$ and $t=(\xi-e) / c$, 
giving

$$
\int_{-1}^{1} \frac{q_{\text {stick }}(t) \mathrm{d} t}{s-t}=F\left(s, \sigma_{0}, c, e\right), \quad|s| \leqslant 1
$$

where $F$ is the normalized form of $G$. The inversion of integral equation (7) is given by

$$
q_{\text {stick }}(t)=-\frac{\sqrt{1-t^{2}}}{\pi^{2}} \int_{-1}^{1} \frac{F\left(s, \sigma_{0}, c, e\right) \mathrm{d} s}{(s-t) \sqrt{1-s^{2}}}, \quad|s| \leqslant 1
$$

providing that the side condition (consistency) is satisfied, namely

$$
\int_{-1}^{1} \frac{F(s, \sigma, c, e) \mathrm{d} s}{\sqrt{1-s^{2}}}=0
$$

The side condition will be enforced first. Integral (9) is evaluated using the Gauss-Chebyshev quadrature [22]. In discretized form it is

$$
\int_{-1}^{1} \frac{F(s, \sigma, c, e) \mathrm{d} s}{\sqrt{1-s^{2}}} \cong \frac{\pi}{n+1} \sum_{i=0}^{n} F\left(s_{i}, \sigma, c, e\right)=0
$$

where

$$
s_{i}=-\cos \left[\frac{(2 i+1) \pi}{2(n+1)}\right], \quad i=0, \ldots, n
$$

The second step of the solution will involve the numerical solution of the integral in equation (8). As the behaviour of $q_{\text {stick }}(t)$ is expected to be bounded at \pm 1 , equation (9) can be rewritten as

$$
\int_{-1}^{1} \frac{\phi(t) \sqrt{1-r^{2}} \mathrm{~d} t}{s-t}=F(s, \sigma, c, e), \quad|s| \leqslant 1
$$

where the regular function $\phi(t)$ can be expressed as a linear combination of Chebyshev polynomials $\left(\mathrm{CP}_{j}\right)$ of the second kind, i.e.

$$
\phi(t) \cong \sum_{j=0}^{m} u_{j} \mathrm{CP}_{j}(t)
$$

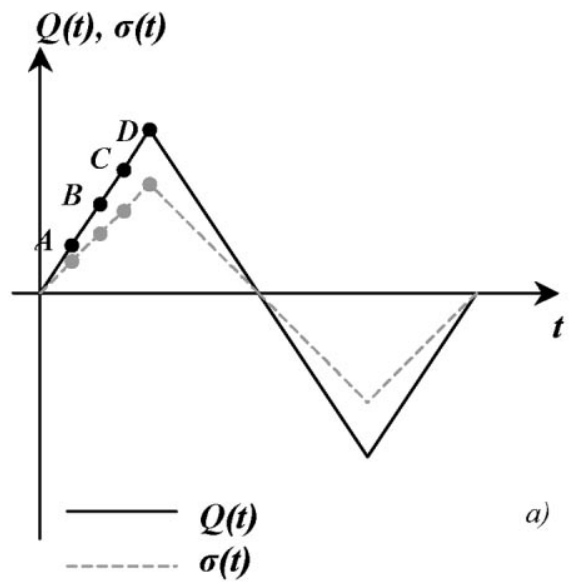

where

$$
\mathrm{CP}_{j}=\frac{\sin \left[(j+1) \cos ^{-1}(t)\right]}{\sin \left[\cos ^{-1}(t)\right]}
$$

Equation (12) can now be replaced by the following numerical representation [23]:

$$
\sum_{j=0}^{m} u_{j} \operatorname{CPI}_{j+1}\left(v_{k}\right) \cong \frac{F\left(v_{k}, \sigma, c, e\right)}{\pi}
$$

where $\mathrm{CPI}_{j+1}$ is the $(j+1)$ th Chebyshev polynomial of the first type and $v_{k}=-\cos [\pi(k+1) /(m+2)]$ (for $k=0, \ldots, m)$.

The last equation needed to solve the problem is found from the imposition of tangential equilibrium at the contact surface:

$$
Q=\int_{-b}^{b} q(t) \mathrm{d} t
$$

Equations (10), (15) and (16) give a system of $m+3$ equations in $m+3$ variables $\left(k_{j}, c, e\right)$, which may be solved using standard routines for treating systems of linear equations.

When the unknown shearing traction distribution, $q(x)$, has been found, the tangential slip displacement, $g(x)$, may be found from

$$
u_{1}(x)-u_{2}(x)=g(x)= \pm \int_{e \pm c}^{x}\left(\frac{A}{\pi} \int_{\text {contact }} \frac{q(\xi) \mathrm{d} \xi}{t-\xi}\right) \mathrm{d} t
$$

where the positive signs are chosen if $x<e-c$ and the negative signs if $x>e+c$.

\subsection{Slip displacement}

Two independent combinations of the dimensionless applied variables characterize the stick-slip regime. These are $Q /(f P)$, where $Q$ is the applied shearing force per unit depth into the page, $P$ is the applied

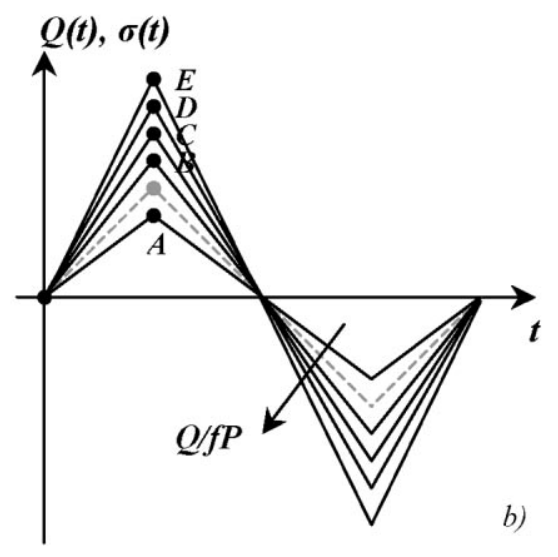

Fig. 3 (a) Loading cycles and (b) combination of possible loading conditions 
normal load per unit depth and $f$ is the coefficient of friction, and $\sigma_{0} a /(f P)$, where $\sigma_{0}$ is the bulk tension present. Combinations of these variables, which give the maximum slip displacement, have been studied. First a flat and rounded contact is considered, where $a / b=0.956$, subject to cyclically varying symmetrical tangential and remote bulk loads varying in phase (see

Fig. 3a), so that

$$
\frac{\mathrm{d} Q}{Q}=\frac{\mathrm{d} \sigma_{0}}{\sigma_{0}}
$$

and hence

$$
\frac{\sigma_{0} a}{Q}=\frac{\sigma_{0} a /(f P)}{Q /(f P)}=\mathrm{constant}
$$

Figure $4 \mathrm{a}$ shows the variation of the shear traction distribution due to increasing values of the load, where the ratio $\sigma_{0} a / Q$ is maintained constant at 4.1 . This value has been chosen because $e+c \simeq b$, and hence the right-hand side of the stick zone is more or less attached to the right-hand contact edge. The receding

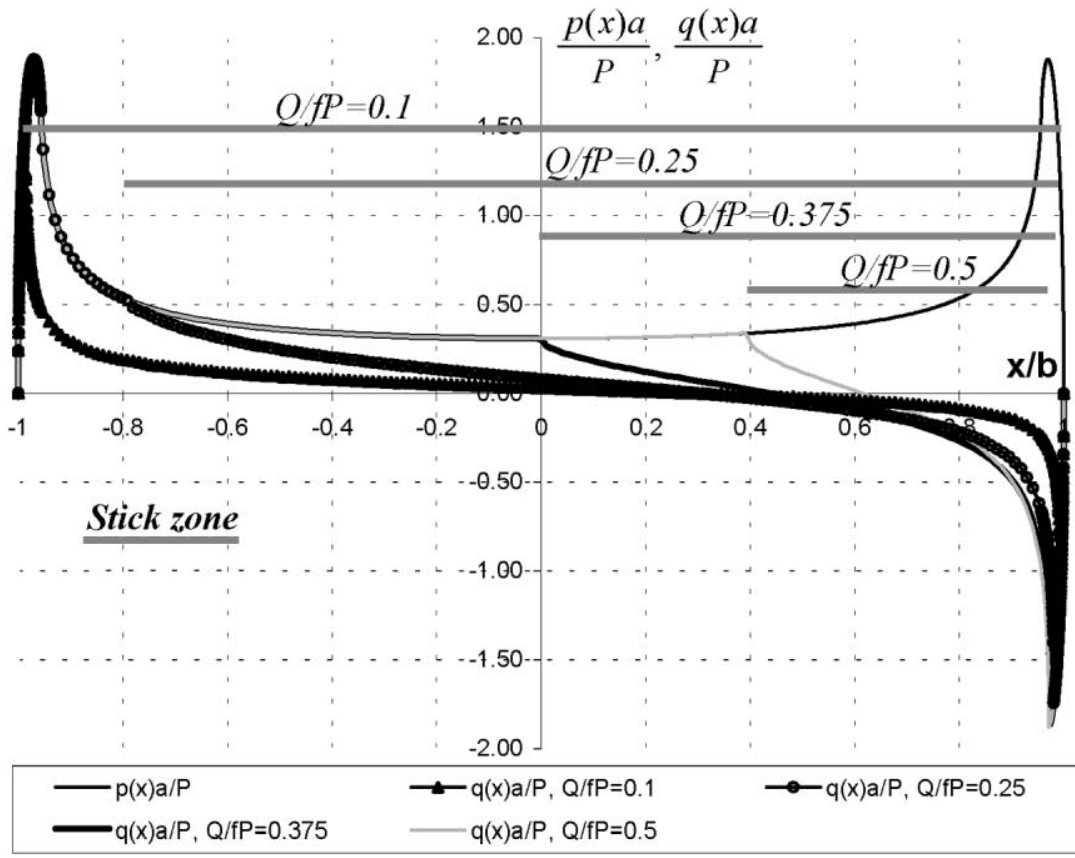

a)

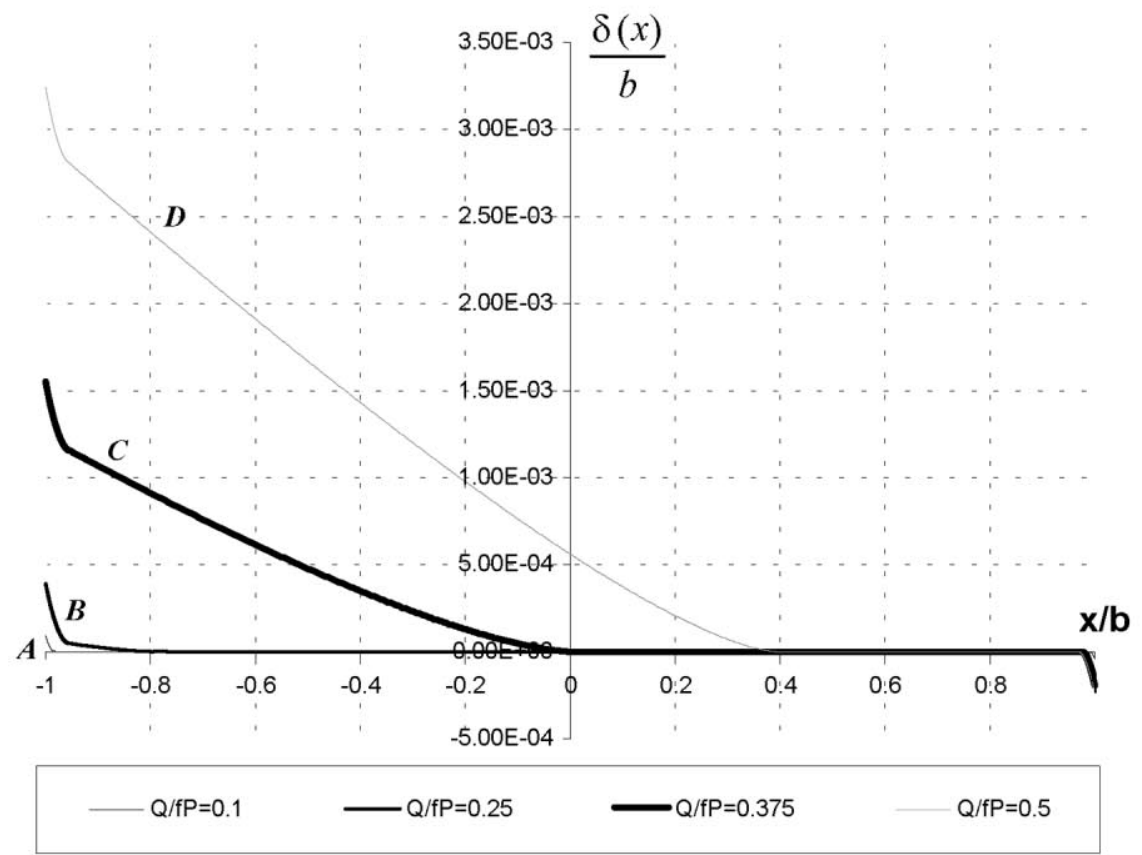

b)

Fig. 4 (a) Pressure, shear tractions and (b) slip displacements during the first quarter of the loading cycle (Fig. 3a) 
stick zone is clearly shown as the load is increased and when the normalized shearing force is 50 per cent of the value needed to cause sliding, the slip zone extends over about 0.6 of the semi-width of the contact patch.

Once the tractions have been computed, it is possible to obtain the slip displacement by integrating equation (17) numerically. Figure $4 \mathrm{~b}$ displays the normalized tangential displacement along the contact surface for the loads used above. As expected, tangential displacements increase monotonically at any given position as the cyclic loads increase (hence the maximum value of slip displacement corresponds to the maximum value of applied load), reaching their peak within the radiused portion of the contact close to the trailing edge of the contact. It should be noted, though, that the variation

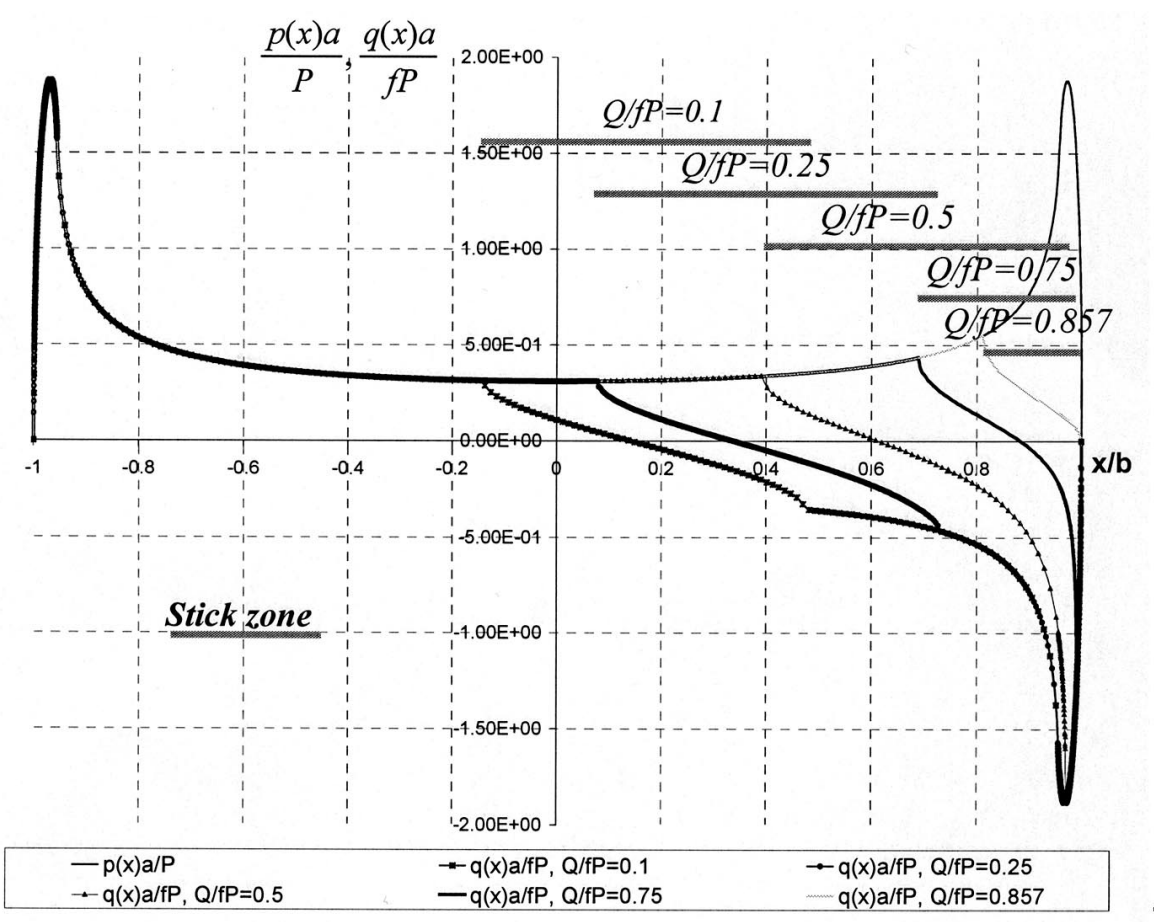

a)

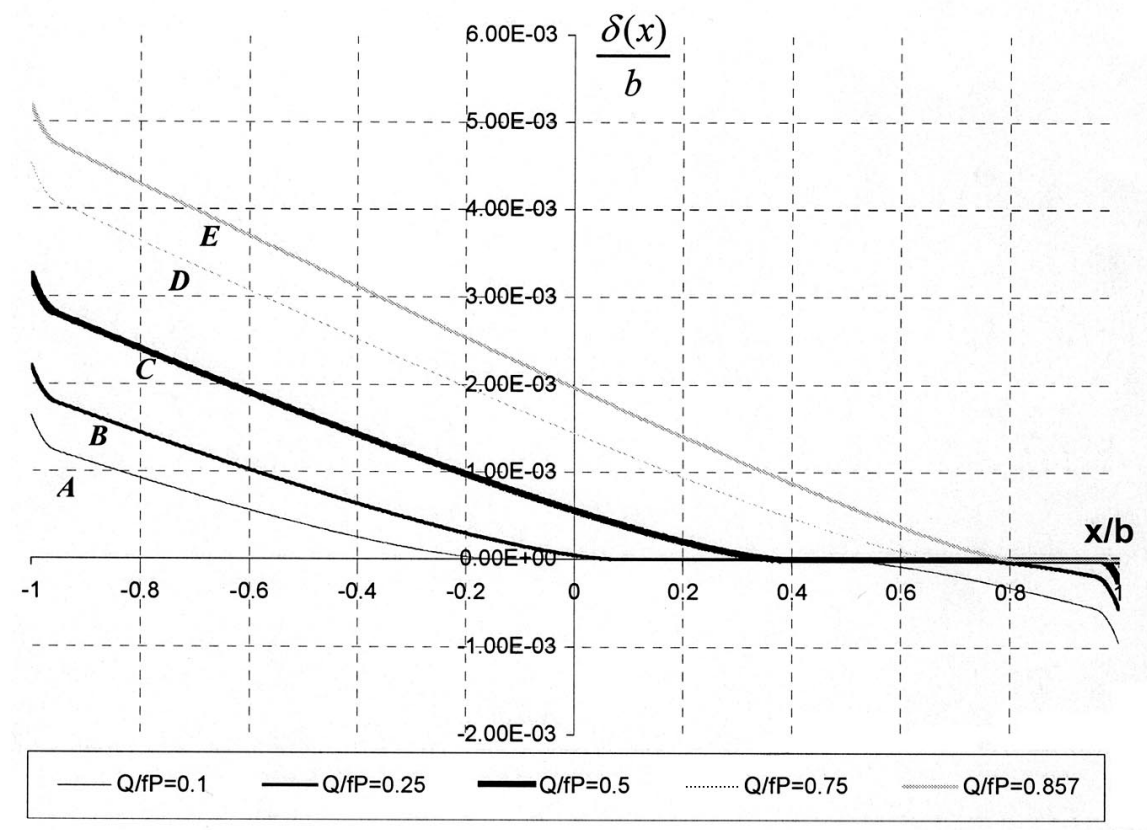

b)

Fig. 5 (a) Pressure, shear tractions and (b) slip displacements at the peak of the loading cycle for different combinations of loads (Fig. 3b) 
of slip displacement with position is strongly affected by the punch geometry. For a given applied load the maximum slip displacement always occurs at the contact edge, as shown in Fig. 4a.

\subsection{Results: partial slip contact}

In section 3.1 the effect of the magnitude of the load on slip displacement was investigated when the ratio of dimensionless shear to tension $\left(\sigma_{0} a / Q\right)$ was kept constant. Here the value of the tension, $\sigma_{0} a /(f P)$, will be fixed and the dimensionless shear, $Q /(f P)$, will be increased. Suppose that $\sigma_{0} a /(f P)=2$; Fig. $3 \mathrm{~b}$ shows schematically the loading state and Fig. 5 shows the corresponding shearing traction distribution, stick-slip regime (a) and slip displacement (b). The maximum slip displacement arises when there is both a shear force and bulk tension present, so that the stick region is vanishingly small and attached to one edge of the contact (curve $\mathrm{E}$ in Fig. 5b). It will immediately be noted that the maximum practicable value for this quantity is $\delta / b \cong 5 \mu \mathrm{m} / \mathrm{mm}$. It follows that, for a contact of half-width $5 \mathrm{~mm}$, which represents quite a large contact in practice, the maximum achievable slip displacement is $\delta \cong 25 \mu \mathrm{m}$.

The effect of such a small relative displacement on the range of stress experienced by a surface particle is very small, and this effect is therefore always negligible under partial slip conditions. Figure 6 shows plots of
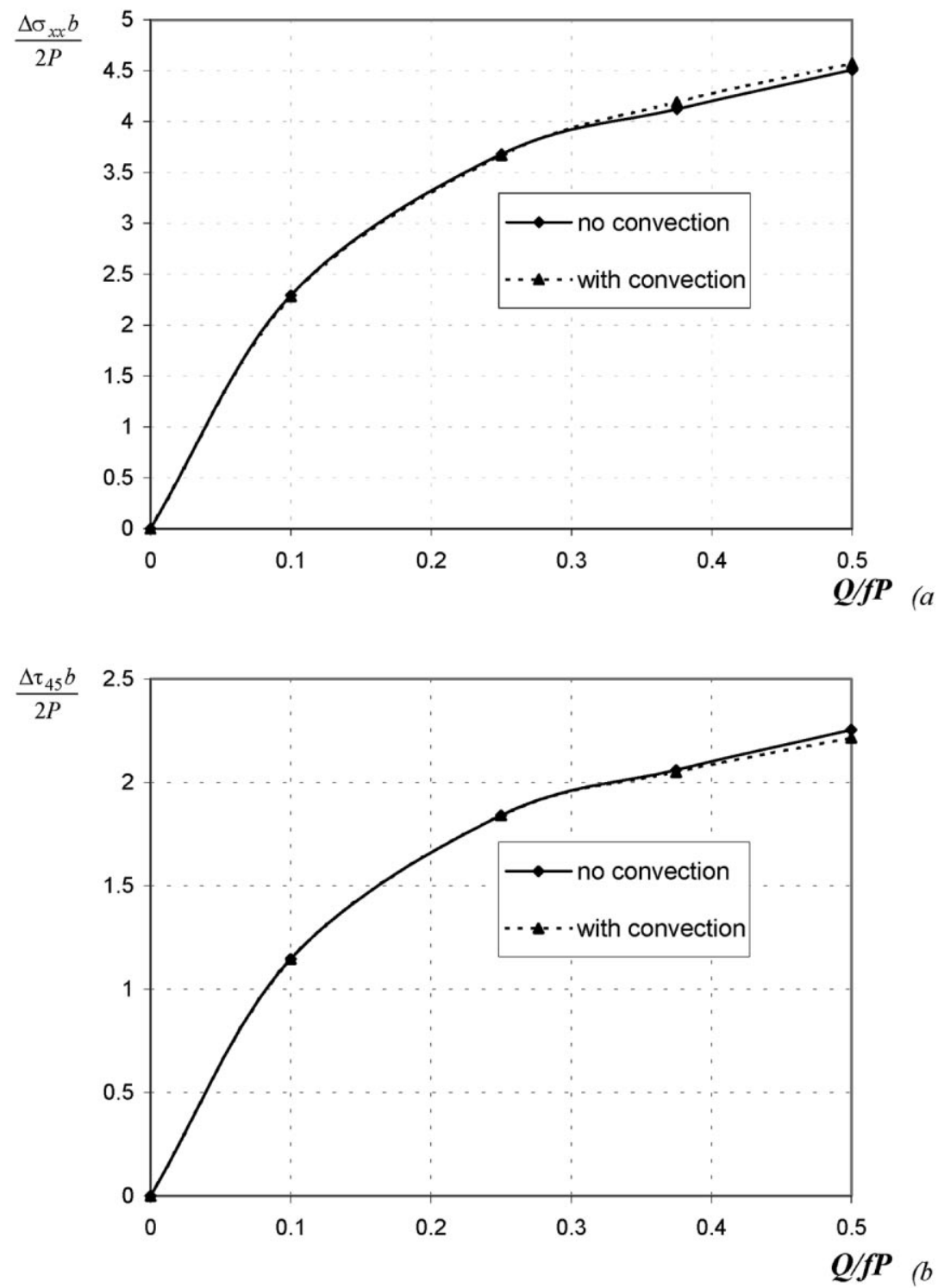

Fig. 6 (a) $\Delta \sigma_{x x} b /(2 P)$ and (b) $\Delta \tau_{45} b /(2 P)$ as functions of $Q /(f P)$ during the loading cycle at the trailing edge of the contact $(x / b=-1)$ 
the key potential crack nucleation controlling quantities, $\Delta \sigma_{x x} b /(2 P)$ and $\Delta \tau_{45} b /(2 P)$ as a function of $Q /(f P)$ during the loading cycle (Fig. 3a) calculated at the trailing edge of the contact $(x / b=-1)$.

\section{SLIDING CONTACT}

The case when the contact suffers a sufficiently large force or imposed slip displacement for sliding to occur will now be considered. Normally the latter will apply because, if the problem were force controlled, the application of a tangential force sufficient to cause sliding would lead to uncontrolled rigid-body displacements. It follows that the slip displacement here is an independent variable, although for the contact to be classified as 'fretting' this should not be too large (say less than $200 \mu \mathrm{m}$ in practical problems), but will certainly exceed the slip displacement associated with partial slip (see the next section for a quantification of this). It is convenient, for generality, to express the slip displacement, $\delta$, as a fraction of the contact half-width, $b$; the effect of $\delta_{\max } / b$ on the range of stress experienced by surface particles in the contacting body (2) needs to be examined. The question then arises about which components of stress have the most important effect on controlling crack nucleation conditions. Here, detailed considerations of this question will be avoided and, instead, three key quantities will be examined: (a) the maximum range of direct stress, $\Delta \sigma_{x x}$; (b) the maximum range of shear stress on a $45^{\circ}$ plane, $\Delta \tau_{45}$; and, lastly, (c) the size of the region swept out by the process (plasticity) zone.

\subsection{Results: sliding contact}

The results found for an example sliding contact are displayed in Fig. 7, where the dimensionless imposed slip displacement has been set to $\delta / b=12 \times 10^{-3}$. Here $\delta$ is the rigid-body displacement and $b$ the contact halfwidth. Figure $7 \mathrm{a}$ displays the range of tension, $\Delta \sigma_{x x} b /(2 P)$, found in a region near the contact corner. Here $\Delta \sigma_{i j}=\sigma_{i j}^{Q_{\max }}-\sigma_{i j}^{Q_{\min }}$, where $Q_{\max }=f P$ and $Q_{\text {min }}=-f P$ (sliding case). In the region of greatest interest, just exterior to the contact, the difference between an analysis allowing for the effect of slip displacement and one ignoring it may be as great as 25 per cent. In the case of the orthogonal shearing stress, $\Delta \tau_{45} b /(2 P)$ (Fig. $7 \mathrm{~b}$ ), the picture is similar. Both on the surface and subsurface, the maximum difference between the two forms of solution in the region of general interest is limited to 15 per cent, and this difference is clearly significant. Lastly, Fig. 7c shows the position of the process zone fronts, as defined by the von Mises yield criterion, for various values of slip displacement. Note that this figure is focused very tightly to the corner of the contact, which is the region where a

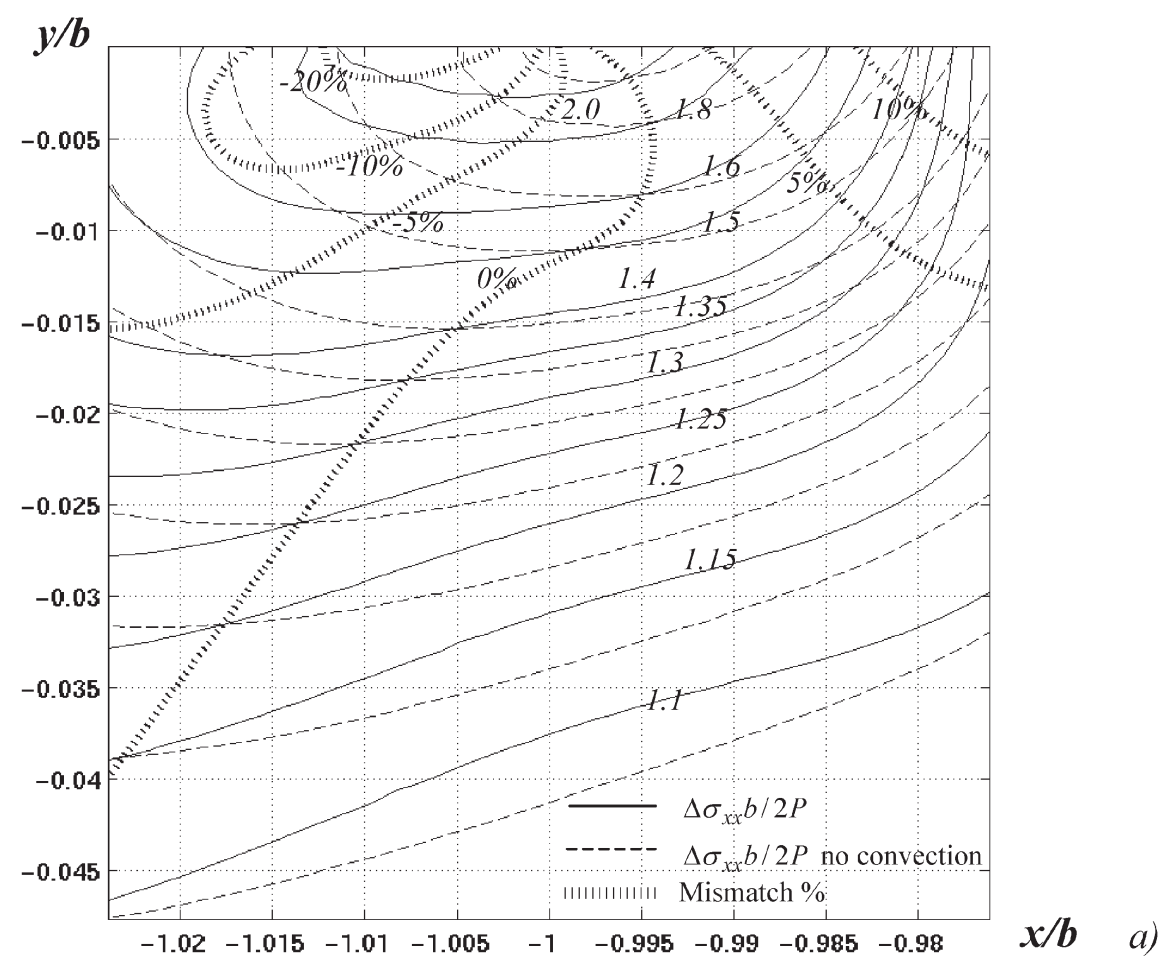

Fig. 7 (continued over) 

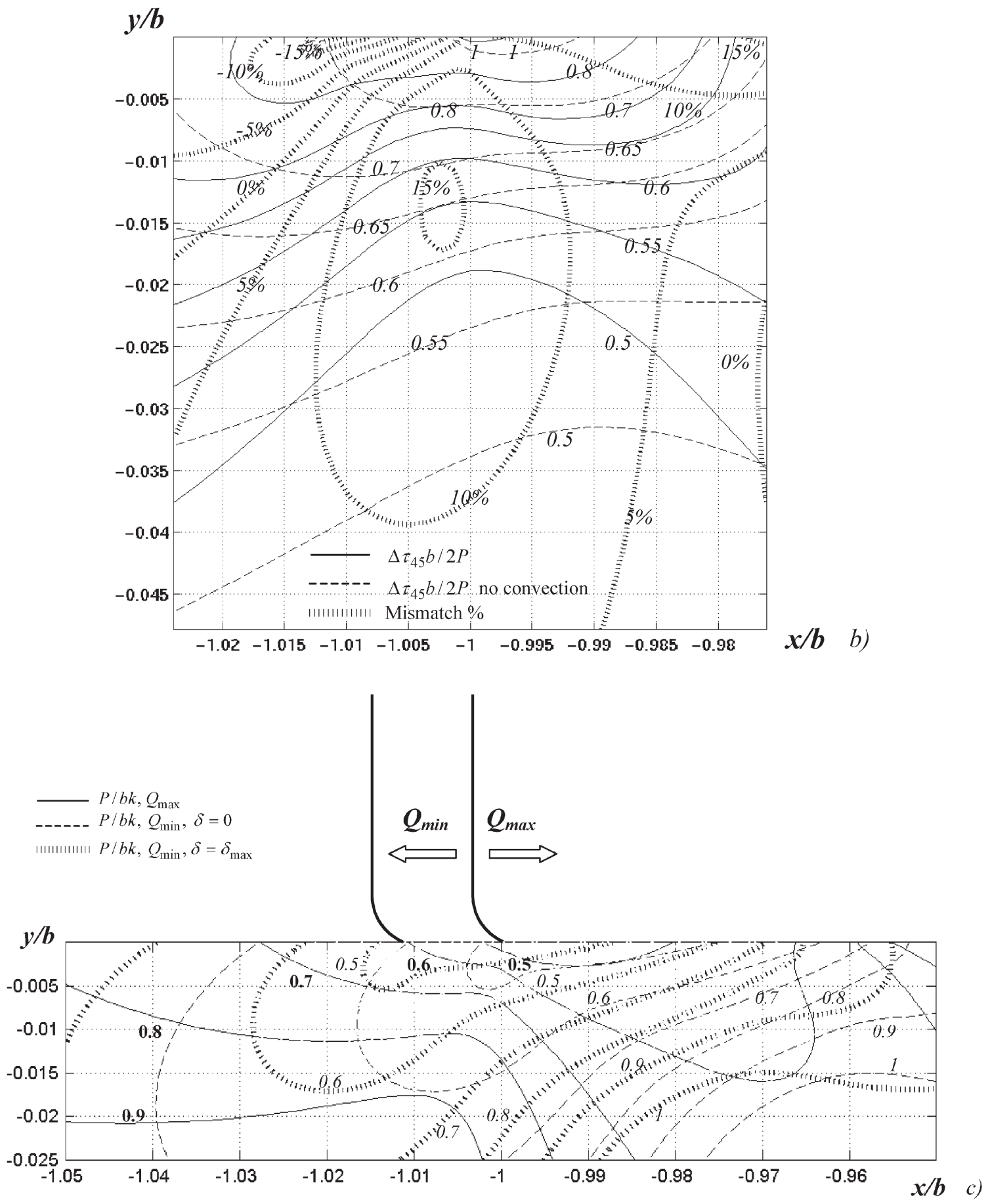

Fig. 7 (a) Range of underlying tension experienced, (b) range of orthogonal shear stress experienced and (c) plastic fronts for various values of $P /(b k)$ at the maximum and minimum shearing loads, allowing for and ignoring 'convection' for a sliding contact, where the dimensionless rigid-body displacement $(\delta / b)$ is set to $12 \times 10^{-3}$

fretting fatigue crack is most likely to nucleate. The plastic zone itself is confined to a region adjacent to the contact surface, and the effect of convection on the total amount of material suffering some plasticity during the loading cycle is investigated. Take, as an example, the behaviour of the component when the dimensionless normal load is $P /(b k)=0.6$. The datum position for the frame of reference is taken to be at the maximum positive shearing force, i.e. when $Q=+f P$. The plastic zone at this point extends to a depth of 
about $0.016 b$ and between $-1.011<x / b<-0.967$. Now suppose that the shearing force is fully reversed, i.e. $Q=-f P$, but that the contacting body is slid to the left by $12 \times 10^{-3} \mathrm{~b}$; the plastic zone is now also shifted to the left by the same amount.

Because the plastic zones at each extreme of loading overlap, it follows that, when convection is allowed for, the total volume of material entering the plastic state at some point during the loading cycle is very much greater (as highlighted by the comparison of the shaded regions in Fig. 7c). As a consequence, the probability of crack nucleation occurring when convection is present is higher.

\section{CONCLUSION}

The negative result that 'convection'-type effects have no effect whatever on the state of stress experienced in the fretting problem studied here is itself an interesting result. It means that, within the partial slip regime and ignoring the effect of wear, so that the initial geometry is assumed to endure, there is no reason to expect slip displacement to have any effect on the stress history experienced. On the other hand, the effect of a relative displacement does have a first-order effect on the stress history when the contacting bodies are in the sliding mode. This suggests that, if the effect of slip displacement on fatigue in partial slip is real, its origin does not lie in the tiny influence that convection has been shown to have in the stress history experienced. Lastly, it should be stated that, although the results presented are geometry-dependent, it would seem very likely that other geometries of the indenter, e.g. the Hertzian contact, would show an even smaller effect than that described here.

Independent experimental corroboration of these findings is not easy to establish. As stated earlier, it is extremely difficult to devise a partial slip experiment in which everything is maintained constant and the slip displacement varied. Indeed, any modification of the load history clearly also modifies the stress state. Therefore, the only possibility would seem to be to change the size of the contact, but this brings an attendant effect of its own!

Thus, the strongest possibility of investigating any 'convection' phenomenon would be in a sliding experiment, albeit at very small displacements. Particular attention would need to be given to the effect of wear on the modification of the surface profile.

\section{ACKNOWLEDGEMENTS}

Daniele Dini wishes to thank Prof. G. P. Demelio, Prof. M. Ciavarella and Dr D. Nowell for fruitful discussion on the development of the numerical techniques and the Department of Trade and Industry and RollsRoyce plc for funding his studies.

\section{REFERENCES}

1 Johnson, K. L. Contact Mechanics, 1985 (Cambridge University Press, Cambridge).

2 Hills, D. A., Nowell, D. and Sackfield, A. Mechanics of Elastic Contacts, 1993 (Butterworth-Heinemann, Oxford).

3 Ciavarella, M. The generalized Cattaneo partial slip plane contact problem. I-Theory. Int. J. Solids Structs, 1998, 35(18), 2349-2362.

4 Jäger, J. Half-planes without coupling under contact loading. Arch. Appl. Mechanics, 1997, 67, 247-259.

5 Swalla, D. R. and Neu, R. W. Influence of coefficient of friction on fretting fatigue crack nucleation prediction. Tribology Int., 2001, 34(7), 493-503.

6 Szolwinski, M. P. and Farris, T. N. Observation, analysis and prediction of fretting fatigue in 2024-T351 aluminum alloy. Wear, 1998, 221(1), 24-36.

7 Araújo, J. A. and Nowell, D. The use of critical plane approaches to predict fretting fatigue initiation life of components subjected to different contact stress fields. In Proceedings of the 6th International Conference on Biaxial/Multiaxial Fatigue, Lisbon, Portugal, 2001.

8 El Haddad, M. H., Topper, T. H. and Smith, K. N. Prediction of non-propagating cracks. Engng Fracture Mechanics, 1979, 11, 573-584.

9 Nowell, D. and Araújo, J. A. Analysis of pad size effects in fretting fatigue using short crack arrest methodologies. Int. J. Fatigue, 1999, 21, 947-956.

10 Sackfield, A., Mugadu, A. and Hills, D. A. The influence of an edge radius on the local stress field at the edge of a complete fretting contact. Int. J. Solids Structs, 2002, 39, 4407-4420.

11 Mugadu, A. and Hills, D. A. A generalised stress intensity approach to characterising the process zone in complete fretting contacts. Int. J. Solids Structs, 2002, 39, $1327-1335$.

12 Mugadu, A., Hills, D. A. and Limmer, L. An asymptotic approach to crack initiation in fretting fatigue of complete contacts. J. Mech. Phys. Solids, 2002, 50, 531-547.

13 Nishioka, K. and Hirakawa, K. Fundamental investigations of fretting fatigue. Bull. Jap. Soc. Mech. Engrs, 1969, 12(5052).

14 Vingsbo, O. and Söderberg, S. On fretting maps. Wear, 1988, 126(2), 131-147.

15 Fouvry, S., Kapsa, P. and Vincent, L. Fretting behaviour of hard coatings under high normal load. Surf. Coatings Technol., 1994, 68-69, 494-499.

16 Fouvry, S., Vincent, L. and Kapsa, P. Analysis of sliding behaviour for fretting loadings: determination of transition criteria. Wear, 1995, 185(1-2), 35-46.

17 Hannel, S., Fouvry, S., Kapsa, P. and Vincent, L. The fretting sliding transition as a criterion for electrical contact performance. Wear, 2001, 249(9), 761-770.

18 Scthayerman, I. Ya. Contact problem of the theory of elasticity. In Gostektheoretizdat, Leningrad, 1949; available from the British Library in an English 
translation by Foreign Technology Division, FTD-MT-2461-70, 1970.

19 Ciavarella, M., Macina, G. and Demelio, G. P. On stress concentration on nearly flat contacts. J. Strain Analysis, 2002, 37(6), 493-501.

20 Navarro, C., Hills, D. A. and Dominguez, J. The effect of corner radius on an asymptotic solution to the fretting of complete contacts. Fatigue Fracture Engng Mater. Structs (in press).
21 Nowell, D. and Hills, D. A. Mechanics of fretting fatigue tests. Int. J. Mech. Sci., 1987, 29(5), 355-365.

22 Conte, S. D. and De Boor, C. Elementary Numerical Analysis, 2nd edition, 1972 (McGraw-Hill, New York).

23 Erdogan, F. M., Gupta, G. D. and Cook, T. S. Numerical solution of singular integral equations. In Methods of Analysis and Solutions of Crack Problems (Ed. G. C. Sih), 1973 (Noordhoff, Groningen, The Netherlands). 